\title{
Frequency Hopping Strategies for Improving Terahertz Sensor Network Performance over Composition Varying Channels
}

\author{
Eisa Zarepour, Mahbub Hassan, Chun Tung Chou \\ School of Computer Science and Engineering
}

University of New South Wales, Sydney, New South Wales 2052, Australia

\{ezarepour, mahbub, ctchou\}@cse.unsw.edu.au

\author{
Adesoji A. Adesina* \\ ATODATECH LLC, Brentwood, CA 94513 USA \\ ceo@atodatech.com
}

\begin{abstract}
The terahertz band is an unlicensed frequency range that is expected to be exploited in the near future for many different types of communications, including wireless communication in nano-scale sensor networks. However, as terahertz band is the resonance frequency of many molecules, communication in this band is severely affected by molecular absorption noise and attenuation. In this paper, we consider a nano-scale terahertz sensor network (TSN) where the chemical composition of the medium varies over time causing absorption for different frequency regions at different times. We propose frequency hopping as a means to overcome the problem of dynamic molecular absorption in composition varying channels. We formulate the frequency selection problem as a Markov Decision Process (MDP), which allows us to adjust the rate of frequency switching for the nano sensors because resource constrained TSN nodes may not be able to switch frequency rapidly. We show that, compared to nonhopping channel selection, frequency hopping can significantly improve capacity and bit error rate when nano sensors have severe power constraints. We propose practically realizable offline policies that obviate the need for observing the channel states, yet perform close to the MDP-based solutions.
\end{abstract}

Keywords-Terahertz nano sensor networks, Compositionvarying channel, Frequency hopping, Markov decision process.

\section{INTRODUCTION}

As we progress toward a future of very small sensors, thanks to advancements in nanotechnology, wireless sensor networks face a new challenge. The small antenna size dictates that these nanosensors operate over very high frequencies, namely the terahertz band [1], [2]. Unfortunately, as terahertz band is the resonance frequency of molecules, communication in this band is severely affected by molecular absorption noise and attenuation.

The entire terahertz band, which spans from $1 \mathrm{THz}$ to $10 \mathrm{THz}$, is likely to be divided into several sub-bands or sub-channels. Only a single sub-channel is then used for a given communication between a transmitter and a receiver at any given time. Interestingly, different species of molecules resonate and absorb terahertz at different frequency sub-bands. Therefore, for a given species composition in the wireless channel, some sub-channels are affected less severely than

\footnotetext{
* This work was done while the author was with the School of Chemical Engineering at University of New South Wales.
}

others. This observation has recently motivated researchers to identify different terahertz sub-channels for different applications of TSNs. For example, Javed and Naqvi [3] have shown that human body and air have different compositions and hence TSNs deployed in these two different environments are optimized with different terahertz sub-channels.

In this paper, we consider a new type of sensor networking environment where the chemical species composition varies over time. For example, TSNs could be potentially deployed inside a chemical reactor to collect information regarding the elementary steps of the reaction. Such detailed molecular level information gathering will allow chemical engineers to observe the process dynamics at a granularity that is beyond the capability of conventional methods. Unfortunately, unlike our daily living environment, the chemical composition inside a reactor varies rapidly due to chain reactions consuming certain molecules and producing others. This time-varying composition means that a sub-channel which has low noise at one time may become very noisy at another time, and vice versa.

We propose frequency hopping as a means to overcome the problem of time-varying channel attenuation and noise. We formulate the sub-channel selection problem at any given time as a Markov Decision Process (MDP), which allows us not only to optimize the communication performance over the entire reaction process, but also to control the rate of channel switching because resource constrained TSN nodes may not be able to switch frequencies rapidly. We show that, compared to conventional non-hopping channel selection, the proposed frequency hopping can significantly improve capacity and bit error rate. Our contributions can be summarized as follows:

- We identify a new application of TSNs, which exhibits composition varying channels. To the best of our knowledge, terahertz communication over composition varying channels has not been studied before.

- We demonstrate that, for composition varying applications, frequency hopping significantly improves the performance of TSNs over the conventional non-hopping channel selection policies. The benefit of frequency hopping is more pronounced when transmission power is severely restricted, which is the case for nano-scale sensor 
networks.

- We propose practical, realizable offline policies that obviate the need for observing the channel states, yet perform close to the MDP-based optimal solutions.

The rest of the paper is structured as follows. TSN and composition varying channels are motivated in Section II. MDP formulation of the frequency hopping problem and offline policies are presented in Section III. Results are analyzed in Section IV. We review related work in Section $\mathrm{V}$ before concluding the paper in Section VI.

\section{TERAHERTZ SENSOR NetworkS}

Nano Sensor Networks (NSNs) [4] is one of the recently introduced TSNs. Nanosensors are tiny motes (nanomotes) made from novel nanomaterials capable of sensing new types of phenomenon at the molecular level. For example, a hydrogen nanosensor was reported in [5], where the optical properties of the palladium layer changes when exposed to hydrogen. Yonzon et al. [6] survey many other types of nanosensors that can be used for chemical and biological sensing. Similarly, significant progress has been made in building nanoactuators that can be used to accomplish some basic tasks at the molecular level by harnessing the interactions between nanoparticles, electromagnetic fields and heat [1], [7]. The next step is to connect these nanosensors into a wireless NSN for wider coverage and control of the environment. NSNs open up the possibility to sense and control important physical processes from the very bottom, right at the molecule level. There are early indications suggesting that such bottom-up approach to sensing and control, which has hitherto not been possible with conventional macro-scale wireless sensor networks, has the potential to radically improve the performance of many applications in medical, biological, and chemical fields [4], [1], [8], [9].

We consider the use of nano sensor nodes to monitor the conditions within a chemical reactor. Due to their very small form factor, these nano sensor nodes will be operating in the terahertz band. In Section II-A, we review the impact of molecular composition on the properties of the terahertz radio channel. After that, in Section II-B, we use a specific chemical process to show that changing composition in the reactor can give rise to a radio channel with different attenuation and noise over time.

\section{A. Terahertz radio channel models}

This section reviews the modelling of terahertz radio channel based on radiative transfer theory presented in [10]. Radio communication is affected by the chemical compositions in two different ways in the terahertz band. First, radio signal is attenuated because molecules in the channel absorb energy in certain frequency bands. Second, this absorbed energy is reradiated by the molecules to create noise in the channel. The use of radiative transfer theory is to model these two effects.

We assume the radio channel is a medium consisting of $N$ chemical species $S_{1}, S_{2}, \ldots, S_{N}$. The effect of each chemical species $S_{i}$ on the radio signal is characterised by its molecular
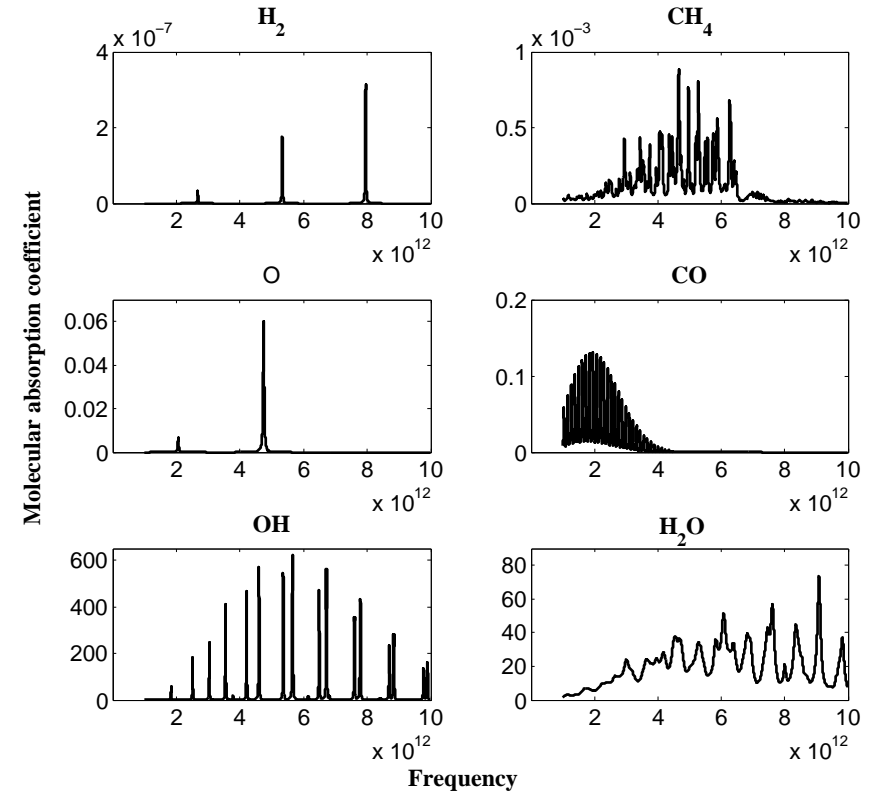

Fig. 1. Molecular absorption coefficient of 6 chemical species. Frequency up to $10 \mathrm{THz}$

absorption coefficient $K_{i}(f)$ of species $S_{i}$ at frequency $f$. The molecular absorption coefficients of many chemical species are available from the HITRAN database [11]. Figure 1 shows the molecular absorption coefficients, up to the frequency of $10 \mathrm{THz}$, for 6 different chemical species at a temperature of $500 \mathrm{~K}$ and pressure of 10atm. The molecular absorption varies across the frequency band. We plot the average molecular absorption over the terahertz band of the six chemical species in Figure 2. It shows that there can be 10 orders of magnitude difference in the molecular absorption coefficients among different chemical species. Of the 6 chemical species, hydroxide $\mathrm{OH}$ and water $\mathrm{H}_{2} \mathrm{O}$ have the highest molecular absorption coefficients.

We consider a single-hop communication via a radio channel in a wireless medium which has time-varying chemical composition. Let $m_{i}(t)$ be the mole fraction of chemical species $S_{i}$ in the medium at time $t$. The medium absorption coefficient $K(t, f)$ at time $t$ and frequency $f$ is a weighted sum of the molecular absorption coefficients in the medium:

$$
K(t, f)=\sum_{i=1}^{N} m_{i}(t) K_{i}(f)
$$

The medium absorption coefficients $K(t, f)$ determines the attenuation and the molecular absorption noise in the radio channel. We first consider attenuation. The attenuation of the radio signal at terahertz is due to be spreading and absorption. Let $A(t, f, d), A_{\text {spread }}(t, f, d)$ and $A_{\text {abs }}(t, f, d)$ be, respectively, the total attenuation, attenuation due to spreading and attenuation due to molecular absorption at time $t$, frequency 


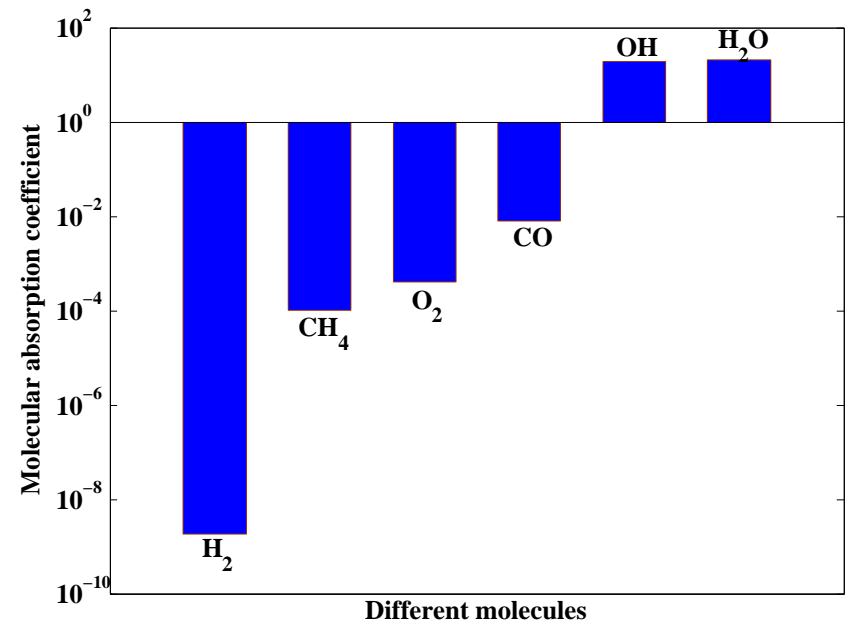

Fig. 2. Molecular absorption coefficient of 6 different chemical species averaged over the terahertz band.

$f$ and a distance $d$ from the radio source. We have

$$
\begin{aligned}
A(t, f, d) & =A_{\text {spread }}(t, f, d)+A_{\text {abs }}(t, f, d) \\
A_{\text {spread }}(t, f, d) & =\left(\frac{4 \pi f d}{c}\right)^{2} \\
A_{\text {abs }}(t, f, d) & =\exp (K(t, f) d)
\end{aligned}
$$

The molecular absorption noise $N_{\mathrm{abs}}(t, f, d)$ which is due to the re-radiation of absorbed radiation by the molecules in the channel is given by:

$$
N_{\text {abs }}(t, f, d)=k_{B} T_{0}(1-\exp (-K(t, f) * d))
$$

where $T_{0}$ is the reference temperature $296 \mathrm{~K}$ and $k_{B}$ is the Boltzmann constant.

Let $U(t, f)$ be the power spectral density of the transmitted radio signal at time $t$ and frequency $f$. The signal-to-noise ratio (SNR) at time $t$, frequency $f$ and distance $d$ is:

$$
\operatorname{SNR}(t, f, d)=\frac{U(t, f)}{A(t, f, d) N_{\mathrm{abs}}(t, f, d)}
$$

Consider a radio channel consisting of two nodes separated by a distance $d$, then at time $t$, the Shannon capacity over a sub-channel from $B_{1}$ to $B_{2} \mathrm{~Hz}$ is:

$$
\text { Capacity }(t, d)=\int_{B_{1}}^{B_{2}} \log _{2}(1+\operatorname{SNR}(\mathrm{t}, \mathrm{f}, \mathrm{d})) \mathrm{df}
$$

\section{B. Composition varying channels}

In our earlier work, we propose to use TSN to improve the proportion of high-value products produced by a chemical reactor. In this paper, we consider the use of TSN to monitor the conditions within a chemical reactor. In order to make our discussion concrete, we have selected the Fischer-Tropsch (FT) synthesis [12] as an example. However, the discussion and methodology in this paper is entirely general.

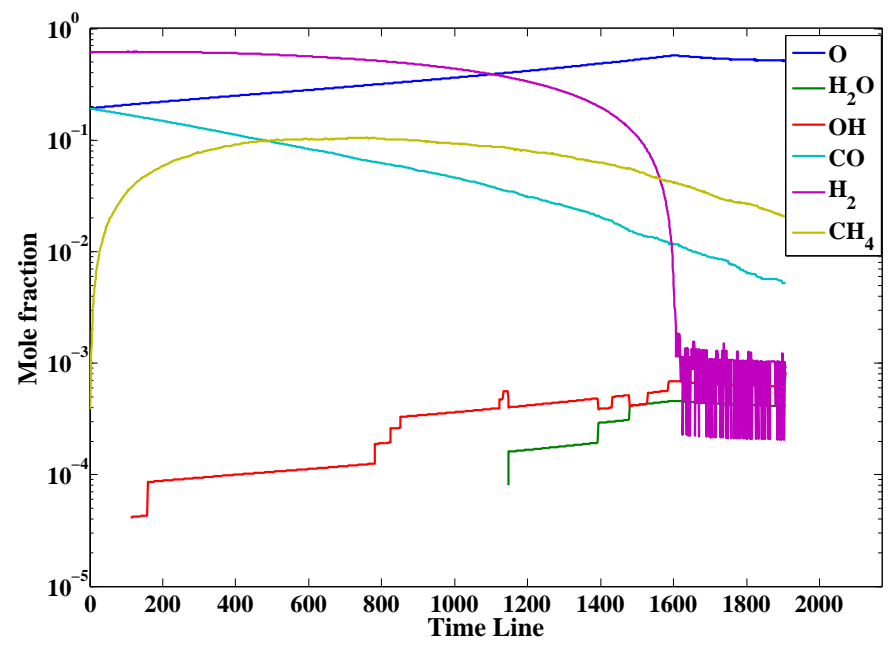

Fig. 3. Evolution of mole fraction of different molecule species during a chemical process. $\mathrm{CO}$ and $\mathrm{H}_{2}$ are consumed while other species are produced gradually.

FT synthesis is a major process for converting natural gas to liquid hydrocarbons in a batch chemical reactor. The reactor starts with a specific amount of carbon monoxide $C O$ and hydrogen $\mathrm{H}_{2}$. Many chemical species are produced and consumed, via many different chemical reactions, during the synthesis. The synthesis stops when no more new products are produced. The contents of the reactor is then emptied to enable the next round of synthesis to begin.

The chemical composition within a FT chemical reactor changes over time. We use the Stochastic Simulation Algorithm (SSA) [13], which is a standard algorithm to simulate chemical reactions (which can be modelled as continuous-time Markov processes), to simulate the FT process with an initial composition consisting of 500 molecules of $\mathrm{CO}$ and 1600 molecules of hydrogen. Figure 3 shows the mole fraction of 6 selected species over time during the course of FT synthesis. It shows that the chemical composition in the reactor changes over time. In particular, we want to focus on the composition of water $\mathrm{H}_{2} \mathrm{O}$ and hydroxide $\mathrm{OH}$ because we know from Figure 2 that these two chemical species have much high molecular absorption coefficient compared with other species. The figure shows that the mole fraction of these chemical species change by one order of magnitude during the course of synthesis.

Note that chemical reactions are inherently stochastic. Figure 3 shows a particular realisation of the Markov process that describes the FT synthesis. There are understandably variations between different realisations but the general behaviour of time-varying composition depicted in Figure 3 remains the same.

The channel modelling in Section II-A shows that the radio channel is affected by the composition of the medium through the medium absorption coefficient $K(t, f)$ (Equation (1)) at time $t$ and frequency $f$. Figure 4 plots the function 
$K(t, f)$ as an heat map (best view in colour) where the $(t, f)$ areas with high medium absorption coefficient $K(t, f)$ are shown as "hot". The "hot" areas correspond to time-frequency regions where the medium has high absorption coefficient and communication between nodes in the TSN will be difficult. The figure shows that different frequency regions become "hot" at different times. Therefore, one can attempt to achieve good communication by choosing a frequency channel that has low $K(t, f)$ over the entire duration of synthesis. We will refer to this as the Best Channel policy. However, we will show in Section IV that this is not the best policy. We will therefore explore the use of frequency hopping in the next section to improve communication performance. We will use the term composition varying channel to refer to radio channels whose medium have changing chemical composition over time.

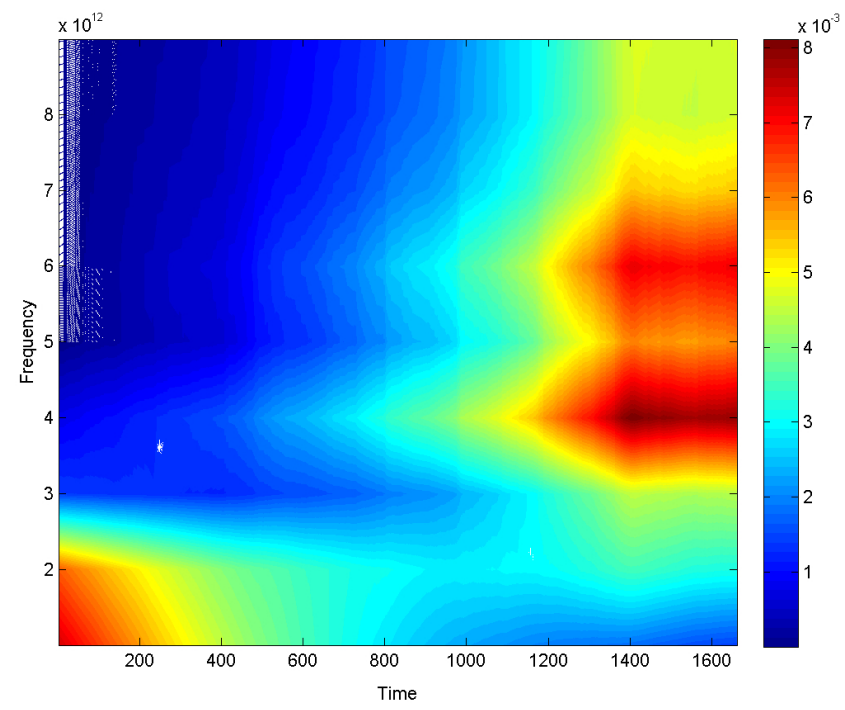

Fig. 4. Frequency-time variation of molecular absorption coefficient $(\mathrm{T}=500$ $\mathrm{K}, \mathrm{P}=10 \mathrm{~atm})$

\section{FREQUENCY HOPPING}

We have demonstrated in Section II-B that composition varying channels exist. In this section, we explore the use of frequency hopping to improve the communication performance of composition varying channels. Three classes of frequency hopping policies will be explored: random, MDP and offline. We first explain the general set-up.

We consider a chemical process with $N$ chemical species $S_{1}, \ldots ., S_{N}$. Let $m_{i}(t)$ be the mole fraction of chemical species $S_{i}$ at time $t$. The state of the reactor at time $t$ is defined by the $N$-vector $\vec{m}(t)=\left[m_{1}(t), \ldots, m_{N}(t)\right]$. In other words, the state of the reactor is defined by the amount of each chemical species in the reactor. We see from Section II-A that $\vec{m}(t)$ also determines the SNR (6) of the radio channel within the chemical reactor.

The chemical composition in the reactor changes over time so $\vec{m}(t)$ is a stochastic process. Chemical reactors can be modelled as Markov processes with state $\vec{m}(t)$. (Appendix A contains an example of modelling a simple chemical process by Markov process.) We use $\mathcal{M}$ to denote the set of all possible compositions in the reactor, so $\vec{m}(t) \in \mathcal{M}$. Let $\vec{m}_{i}$ and $\vec{m}_{j}$ denote two possible states in $\mathcal{M}$, then the transition rate $R\left(\vec{m}_{i}, \vec{m}_{j}\right)$ from $\vec{m}_{i}$ to $\vec{m}_{j}$ is in fact the rate of chemical reactions to change the composition from $\vec{m}_{i}$ to $\vec{m}_{j}$.

We will discretize the continuous-time Markov process using a sufficiently small sampling interval $\Delta$ to turn it into a discrete-time Markov chain. We will henceforth assume that time is discrete and express it in terms of time slots of duration $\Delta$. We index time slots by $h=1,2, \ldots$ and the $h$-th time slot is the time interval $[(h-1) \Delta, h \Delta]$. The set of possible states of the Markov chain is also $\mathcal{M}$. The transition probability from state $\vec{m}_{i}$ to state $\vec{m}_{j}$, will be denoted by $P\left(\vec{m}_{i}, \vec{m}_{j}\right)$, is $R\left(\vec{m}_{i}, \vec{m}_{j}\right) \Delta$. Note that with a sufficiently small $\Delta$, we can assume that $\vec{m}(t)$ is constant over each time slot. Given this assumption, we also use $\vec{m}(h)$ to denote the state in time slot $h$. We also assume that packet transmission can be completed within a time slot.

We assume that the terahertz band extends from $1-10 \mathrm{THz}$ as the recently proposed Graphene based nano-antenna radiates in this range [2]. We use $B$ to denote the bandwidth of the entire band, i.e. $\mathrm{B}=9 \mathrm{THz}$. We divide the entire band into $K$ frequency sub-channels of equal bandwidth $\frac{B}{K}$. We denote the set of frequency sub-channels by $\mathcal{K}=\{1,2, \ldots, K\}$.

The aim of a policy is to determine, for each time slot $h$, the frequency sub-channel $C(h) \in \mathcal{K}$ that the TSN nodes should use during the time slot. We assume the nodes decide on which sub-channel to use before a time-slot and performs the switching at the beginning of the slot.

\section{A. Random frequency hopping}

A well-known frequency hopping policy is random frequency hopping (R-FH). In our case, R-FH means that in each time slot $h$, the sub-channel to be used $C(h)$ is randomly chosen from the set $\mathcal{K}$. Practical implementation means that the sub-channels are computed from a pseudo-random number generator. The advantage of R-FH is that no channel observations are required. However, R-FH does not make use of any knowledge of the state of the channel or the chemical reactor. There is a chance that the sub-channel selected for a time slot has a high medium absorption coefficient, which results in low SNR and poor quality radio communication.

\section{B. Markov Decision Process (MDP)}

We use MDP to compute a frequency hopping policy that improves SNR but at the same time limits the number of switchings. In order to define the MDP, we recall that the chemical composition in the reactor evolves according to a discrete time Markov chain. The formulation of the MDP is as follows:

- States The state $s$ of the MDP is the ordered pair $s=$ $(\vec{m}, k) \in \mathcal{M} \times \mathcal{K}$

- Actions The set of actions at each state is $\mathcal{K}$. 
- Transition Probability The transition probability from state $\left(\vec{m}_{i}, k_{i^{\prime}}\right)$ to state $\left(\vec{m}_{j}, k_{j^{\prime}}\right)$ under action $a \in \mathcal{K}$ is $P\left(\vec{m}_{i}, \vec{m}_{j}\right)$ if $a=k_{j^{\prime}}$, otherwise zero. This means that if action $a$ is used, then the next state uses channel $a$ for communication.

- Reward function As the overall goal of our optimisation is to improve SNR, we use the improvement of SNR as the reward. We use $\widehat{\operatorname{SNR}}\left(\vec{m}_{i}, k_{i^{\prime}}, d\right)$ to denote the mean SNR when channel $k_{i^{\prime}}$ is used for composition $\vec{m}_{i}$ and nodes separated by a distance of $d$. We define the reward function $R\left(s_{i}, s_{j}\right)$ for moving from state $s_{i}=\left(\vec{m}_{i}, k_{i^{\prime}}\right)$ to state $s_{j}=\left(\vec{m}_{j}, k_{j^{\prime}}\right)$

$$
R\left(s_{i}, s_{j}\right)=\widetilde{\operatorname{SNR}}\left(\vec{m}_{j}, k_{j^{\prime}}, d\right)-\widetilde{\operatorname{SNR}}\left(\vec{m}_{i}, k_{i^{\prime}}, d\right)
$$

- Penalty The role of penalty is to reduce the number of switchings. The penalty $P\left(s_{i}, s_{j}\right)$ of moving from state $s_{i}=\left(\vec{m}_{i}, k_{i^{\prime}}\right)$ to state $s_{j}=\left(\vec{m}_{j}, k_{j^{\prime}}\right)$ is $L\left(s_{i}, s_{j}\right)=\alpha$. This means that there is a penalty every time the channel is switched. In our anlsysis, we assume $\alpha=1$ but it could be considered as a design parameter.

- Revenue The revenue function will be

$$
\left.R\left(s_{i}, s_{j}\right)-w L_{(} s_{i}, s_{j}\right)
$$

where $w$ is a weight on the penalty.

Although the MDP policy is optimal in a certain sense, it requires the TSN nodes to be able to observe $\vec{m}(h)$ which is the composition of the channel at time slot $h$, but this is not possible. We therefore explore the use of offline policy which does not require state observations.

A special case of the MDP policy is obtained by choosing penalty weight $w=0$. This results in a greedy policy where in each time slot $h$, the TSN nodes uses the sub-channel in $\mathcal{K}$ that has the maximum SNR. In this case, the number of switchings is not limited and it gives the maximum achievable overall SNR for all policies. On the other hand, if we choose $w$ to be large, then hopping between sub-channels is discouraged.

Different values of $w$ will be used in the numerical evaluation in Section IV. We will call the policy obtained by MDP by setting $w=0$, the MaxSNR policy because it can achieve the maximum average SNR possible, though of course at the expense of higher number of switchings.

\section{Offline policies}

The offline policy can be considered to be a pre-planned or open-loop policy. The offline policy specifies a sequence $k_{1}, k_{2}, k_{3}, \ldots$ where $k_{h} \in \mathcal{K}$ and $k_{h}$ is the channel to be used by the TSN nodes at time slot $h$.

We propose two offline policies. These two policies are based on, respectively, the most probable composition $\vec{m}_{\mathrm{MP}}(h)$ and the average composition $\vec{m}_{\mathrm{AVG}}(h)$, in time slot $h$. We refer to these two offline policies as MCOP (Most probable Composition Offline Policy) and ACOP (Average Composition Offline Policy). For the time being, we will assume that $\vec{m}_{\mathrm{MP}}(h)$ and $\vec{m}_{\mathrm{AVG}}(h)$ are available and we will describe later on how they can be computed.
1) Obtaining the offline policies: We first describe the algorithm to obtain the offline policy using the most probable composition MCOP. The algorithm is based on MDP. Let $\mathcal{M}_{\mathrm{MP}}=\left\{\vec{m}_{\mathrm{MP}}(1), \vec{m}_{\mathrm{MP}}(2), \vec{m}_{\mathrm{MP}}(3), \ldots\right\}$. We consider a Markov chain with $\mathcal{M}_{\mathrm{MP}}$ as the set of all possible states and the transition probability from $\vec{m}_{\mathrm{MP}}(h)$ to $\vec{m}_{\mathrm{MP}}(h+1)$ is 1 while all other transition probabilities are zero. We define a MDP in the same way as Section III-B where we replace the set of states of MDP by $\mathcal{M}_{\mathrm{MP}} \times \mathcal{K}$. We solve this MDP to obtain MCOP.

The offline policy based on average composition ACOP is similar to that based on the most probable composition. The method is the same as above except we replace $\vec{m}_{\mathrm{MP}}(h)$ by $\vec{m}_{\mathrm{AVG}}(h)$. Now we explain how $\vec{m}_{\mathrm{MP}}(h)$ and $\vec{m}_{\mathrm{AVG}}(h)$ can be calculated.

2) Calculation of $\vec{m}_{\mathrm{MP}}(h)$ : It is in principle possible to calculate $\vec{m}_{\mathrm{MP}}(h)$ analytically from the Markov chain by solving the Chapman-Kolmogorov equation of the Markov chain. However, this is practically impossible because the size of the state space $\mathcal{M}$ is exponential in terms of the number of molecules in a reactor, which is generally large. We therefore choose to use SSA simulation to obtain the most probable composition at each time slot. The procedure is:

(a) Run SSA $1000(=\mathrm{J})$ times. Let $\vec{m}_{i}(h)$ be the composition in time slot $h$ for the $i$-th simulation run. Let $H_{i}$ denote the last time slot of simulation run $i$.

(b) Let $H_{\min }=\min \left(H_{1}, \ldots, H_{J}\right)$.

(c) Set $\vec{m}_{i}(h)=0$ for all $h>H_{\text {min }}$

(d) For each $h \in\left[1, H_{\min }\right]$, find the most frequent distribution from $\left\{\vec{m}_{1}(h), \ldots, \vec{m}_{J}(h)\right\}$ and set that equal to $\vec{m}_{\mathrm{MP}}(h)$. If multiple compositions are most frequent, set $\vec{m}_{\mathrm{MP}}(h)$ to one of them randomly.

3) Calculation of $\vec{m}_{\mathrm{AVG}}(h)$ : We used a similar procedure to generate $\vec{m}_{\mathrm{AVG}}(h)$ via extensive SSA simulations. The steps are identical to those used for computing $\vec{m}_{\mathrm{MP}}(h)$ except step (d) is replaced by (d')

(d') For each $h \in\left[1, H_{\min }\right]$, calculate

$$
\begin{gathered}
\vec{m}_{\mathrm{AVG}}(h)=\frac{\sum_{i=1}^{J} \vec{m}_{i}(h)}{J} \\
\text { IV. RESULTS }
\end{gathered}
$$

\section{RESUlTS}

In this section, we study the performance of the proposed frequency hopping schemes. We assume the chemical reactor runs an FT process starting with 500 carbon monoxide molecules and 1600 hydrogen atoms. We assume a conventional FT reactor with equal kinetic constants of 10 for all possible reactions except water formation reactions that has been considered as 0.07 and 2 for $\mathrm{O}+\mathrm{H} \longrightarrow \mathrm{OH}$ and $\mathrm{OH}+\mathrm{H} \longrightarrow \mathrm{H}_{2} \mathrm{O}$, respectively. The chemical production continues until no more new chemicals can be produced. Molecular absorption coefficient of the chemical species produced within the reactor is obtained from the HITRAN database [11] for a temperature of $500 \mathrm{~K}$ and pressure of 10 atmospheres. We follow the procedure in Section II-A to compute the medium absorption coefficient, attenuation, molecular noise 
and SNR. The capacity for each sub-channel is obtained using the Equation(7) that $B_{1}$ and $B_{2}$ are the lowest and highest frequency of the sub-channels, respectively. We solve the MDP optimization problems using the MDP solver in MATLAB [14].

We first analyze the simple case of two sub-channels to illustrate the benefit of frequency hopping in a composition varying environment. We then follow it up with a more general case of $N$ sub-channels.

\section{A. Two sub-channels}

We divide the terahertz band of $1-10 \mathrm{THz}$ into 2 subchannels of $4.5 \mathrm{THz}$ each, where sub-channels 1 and 2 refer to $1-5.5 \mathrm{THz}$ and $5.5-10 \mathrm{THz}$, respectively. Following [15], we employ a uniform power spectral density (PSD) to compute the average SNR of the two sub-channels during the course of the FT synthesis ${ }^{1}$. In other words, for equation (6), we assume $U(t, f)=\frac{P_{\text {total }}}{W}$, where $P_{\text {total }}$ is the total signal power available (power budget) and $W$ is the channel bandwidth.

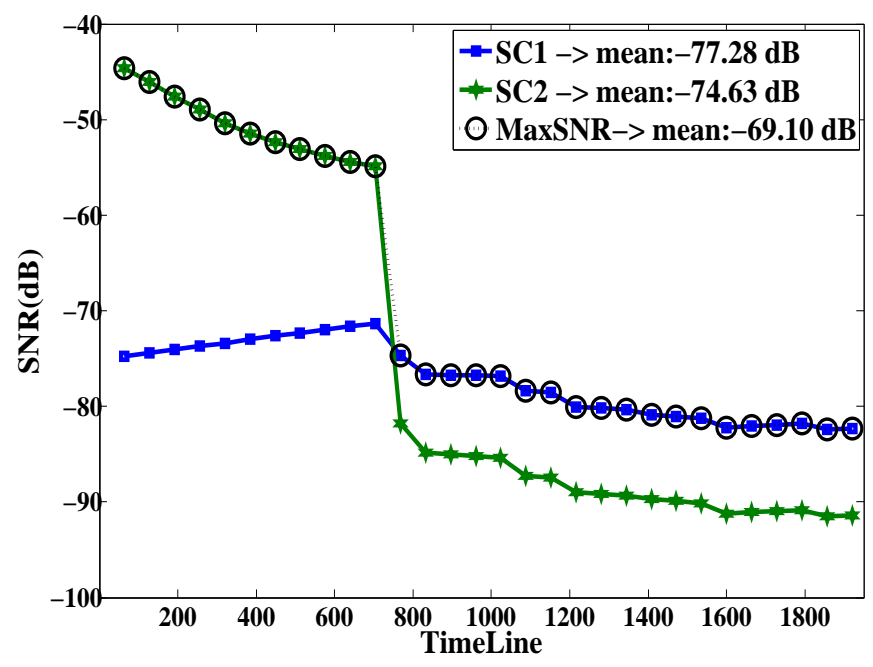

Fig. 5. SNR over time for using SC1, SC2 and MaxSNR.

Figure 5 plots the SNR against time for the two subchannels. We see that initially SC2 was performing better than $\mathrm{SC} 1$, but the situation is reversed after about 800 time slots. This happens because the amount of hydroxide $O H$ in the medium starts to increase as time approaches 800 (see Figure 3 ). Given the absorption spectral density of $\mathrm{OH}$ (see Figure 1), we find the average absorption coefficient for $\mathrm{SC} 1$ and $\mathrm{SC}$, respectively, for $\mathrm{OH}$ is 13.74 and 24.4. Therefore, when $\mathrm{OH}$ starts to increase around time $800, \mathrm{SC} 2$ gets affected more severely than SC1. This clearly shows that selection of either of the two sub channels for the entire FT process would yield non-optimal solution. The MaxSNR solution can be achieved if channel switching (frequency hopping) is allowed, in which

\footnotetext{
${ }^{1}$ We have not chosen to use water filling because the limited capability of TSN motes may not allow them to shape the power spectrum of the input
}

case the system could employ SC2 in the beginning and switch to $\mathrm{SC} 1$ at time 800 .

Let us analyze the benefit of $\mathrm{FH}$ compared to the best channel policy. For SC1 and SC2, the average SNRs over the entire duration of the synthesis are $-77.3 \mathrm{~dB}$ and $-74.6 \mathrm{~dB}$, respectively. The best channel policy would therefore select SC2 and achieve an average SNR of $-74.6 \mathrm{~dB}$. However, the proposed frequency hopping solution, which would employ SC2 up to time 800 and then switch to SC1 afterwards, would achieve an average SNR of $-69.10 \mathrm{~dB}$ over the entire reaction process. This leads to an improvement of $5.5 \mathrm{~dB}$ for the FH over the conventional non-hopping solution.

\section{B. Higher number of sub-channels}

Now we investigate the performance of FH in more general settings. We consider up to 50 available sub-channels of equal bandwidth by dividing the entire terahertz band into 2 to 50 subchannels and compute the average SNR performance (see Figure 6) for different channel selection policies for a power budget of $1 \mathrm{pW}$ and a distance of $1 \mathrm{~m}$ between the transmitter and the receiver. As expected, with increasing number of sub-channels, the SNR increases irrespective of the policy, because the total power is distributed over a smaller range of frequencies boosting the signal power. The most important result is that the MaxSNR, which is obtained when MDP is employed without any penalty for channel switching, improves SNR by around $10 \mathrm{~dB}$ and $20 \mathrm{~dB}$ in comparison with Best Channel and Random policy, respectively, irrespective of the number of sub channels.

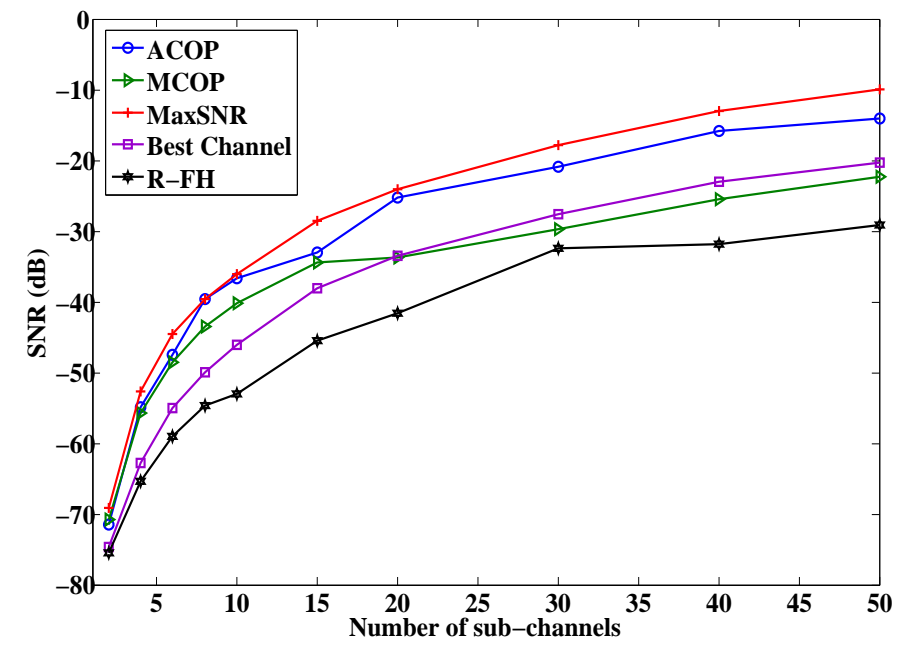

Fig. 6. Achievable SNR via different policies versus number of sub-channels

We observe that the ACOP offline policy achieves reasonably close to the MaxSNR. This is an encouraging outcome because it means that the theoretical benefits of FH can indeed be realized reasonably well in practice. The reason it performed so well is because, although we observed different compositions for a given time slot at different runs of the 


\begin{tabular}{|l|l|l|}
\hline Penalty $(w)$ & Number of Switches & Average SNR(dB) \\
\hline 0 & 3 & -44.97 (MaxSNR) \\
\hline $3-24$ & 2 & -47.30 \\
\hline 27 & 1 & -52.50 \\
\hline 30 & 0 & -54.97 (Best Channel) \\
\hline
\end{tabular}

TABLE I

EFFECT OF CHANNEL SWITCHING PENALTY ON THE PERFORMANCE OF FH $($ POWER $=1 \mathrm{PW}$, DISTANCE $=1 \mathrm{M})$

SSA simulation, the variance in chemical composition at each time is not high. Therefore, the absorption coefficient, and the resulting SNR, of the average composition worked as a good predictor of the channel.

MCOP, however, fails to achieve a good performance (it is worse than the Best Policy in some cases). The reason for the poor performance is the lack of a "highly likely composition" for a given time slot. From 1000 runs of the SSA simulation, we find that the frequency counts of each distinct composition is close to each other. Therefore using one of the composition for channel prediction yields a poor channel estimation.

Next, we investigate the performance of FH when restrictions are imposed on the rate of channel switching. This is achieved by introducing penalty in MDP. For the case of 6 available sub-channels, Table I presents the effect of increasing penalty and the number of channel switching on the performance of FH. We find that there are a maximum of three switches for this reaction process. By allowing just one channel switch, the SNR can be increased from $-54.9 \mathrm{~dB}$ to $-52.5 \mathrm{~dB}$, an improvement of $2.33 \mathrm{~dB}$. With one extra switch, the SNR improves by a further 5.2dB. Finally, the third switch yields another $2.47 \mathrm{~dB}$ improvement. The incremental gain in SNR, which can be achieved by adjusting the penalty, implies that the MDP framework is an effective tool to control the trade off between communication performance and channel switching overhead in the TSN.

The SNR performance gains would ultimately translate to gains in capacity and BER. Figure 7.a shows that FH can improve the capacity by about 2 orders of magnitude compared with Best Channel policy.

Figure 7.b shows the bit-error rate (BER) for the MaxSNR and Best Channel policies. The BER is computed by assuming Pulse Amplitude Modulation over an additive white noise where the magnitude of the white noise comes from the average magnitude of molecular absorption noise over the channel. This BER is notably not exact but for indication purpose only. The achievable BER of the frequency hopping is expected to be between that of MaxSNR and Best Channel policies.

Finally, we study the benefit of $\mathrm{FH}$ as a function of available power budget. Figure 8 shows that the performance gain of FH compared to the Best Channel policy is more pronounced when the available power is more limited. This is intuitive because the Best Channel policy can improve its performance with higher power reducing the difference with FH. However, this also means that FH is an effective strategy to improve the

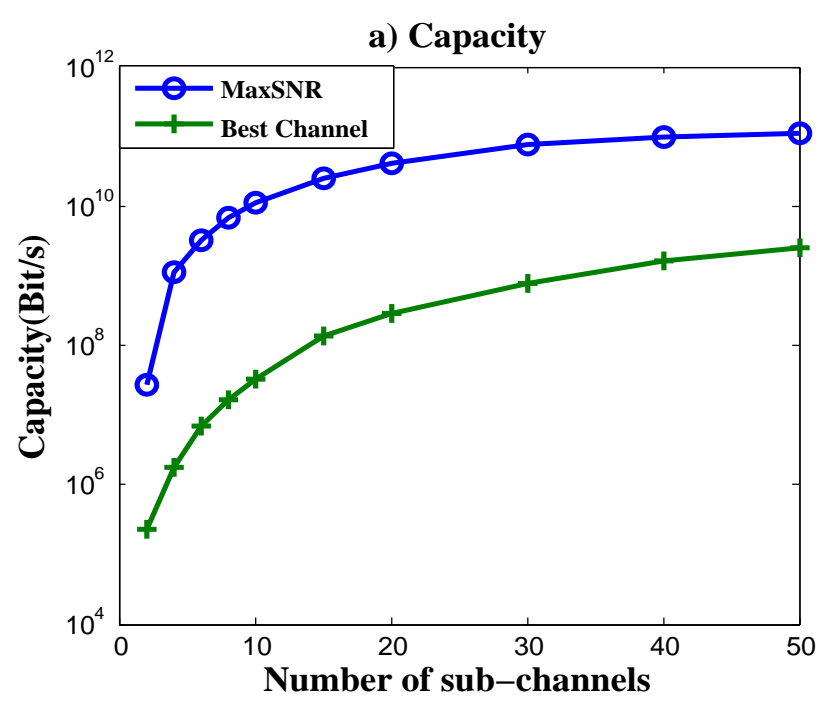

b) Bit Error Rate (BER)

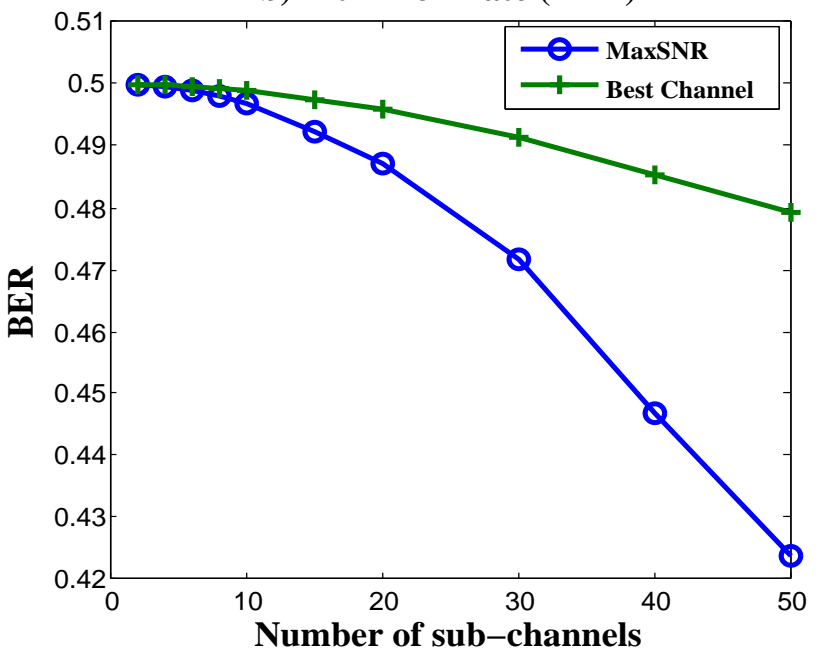

Fig. 7. Capacity and BER comparison between MaxSNR and Best Channel (power $=1$ pico $\mathrm{W})$.

communication performance of nano-scale TSNs, which have a very limited power budget.

\section{RELATED WORK}

It is well known that adaptive frequency hopping (FH) systems can improve communication performance by selecting frequencies with the least interference [16]. The history of FH went back to the Second War when protection against eavesdropping and jamming was needed and such protection could be provided by utilizing frequency hopping. However, these days FH is employed for a wide range of applications such as radar [17], [18], microwave imaging [19]. In general, 

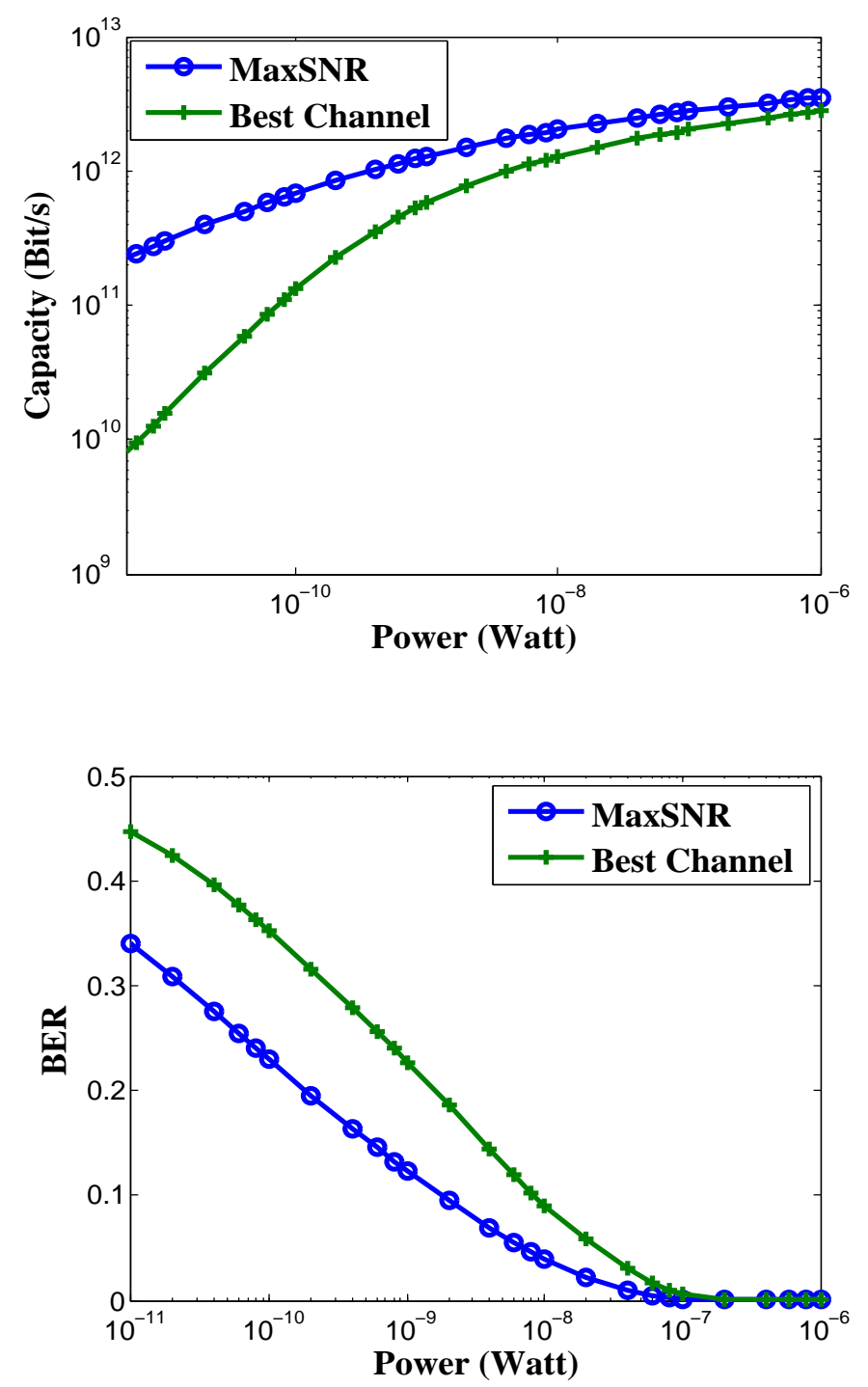

Fig. 8. Performance of MaxSNR and Best Channel policies over different power levels.

spread spectrum (SS) radio technology provides robustness against various kinds of interference and multipath distortion [20]. In particular, frequency hopping spread spectrum (FHSS) has been used in wireless personal area networks (WPANs), such as Bluetooth technology, to mitigate the interference between wireless local area networks (WLANs) and Bluetooth [21].

While the idea of FHSS has been implemented in our work as a benchmark, unlike FHSS and other random FH schema which generate the hopping sequence in a pseudorandom way, we make use of the knowledge of the chemical reactions to design a good hopping sequence. Generating random sequence of the FHSS is another category of the research in this area which aims to generate more efficient sequence. For example, authors in [22] propose few sets of frequency hopping sequences using a class of irreducible cyclic codes. Similar to [23], they aim to exploit different random sequences to reduce the ratio of collision between different simultaneous transmitters. However, they do not take the channel information into account to produce the hopping sequence. Our approach in this paper differs from these methods in a few different aspects. Firstly, we use a priori knowledge about the channel condition to derive a near optimum hopping sequence and we show this priori knowledge can considerably improve the performance of the system. Secondly, the wireless channel variation is caused by changing molecular composition in a chemical reactor.

There is a very limited research conducted on using narrow terahertz channels for wireless NSNs. In a recent work, Javed and Naqvi [3] propose some beneficial narrow sub-channels for NSNs in different environments with different compositions such as human body and air. They divide the terahertz band channel into a number of sub-channels and in each type of medium, they investigate the performance of sub-channels. Finally, they suggest the best one to use for communication. However, the scenario considered in this paper is different because our aim is to monitor the conditions within a chemical reactor. Due to the production and consumption of different chemical species in the chemical reactor over time, the channel conditions change constantly. We therefore propose frequency hopping to improve the overall communication performance.

\section{CONCLUSION}

We have proposed and evaluated frequency hopping as a means to overcome the problem of dynamic molecular absorption in composition varying TSN channels. We have shown that when there is no penalty or cost implications for dynamically switching channels, TSN performance can be improved significantly compared to the conventional nonhopping channel selection policies. With restrictions imposed on channel switching, the performance of frequency hopping reduces linearly. We have shown that MDP is an effective tool to optimize frequency hopping with control over the number of channel switches. A drawback of the MDP solution is that it requires the nano sensors to observe the composition of the channel, which is impractical. We have proposed offline policies that obviate the need for observing the channel states, yet perform close to the MDP-based solutions.

In this paper, we have addressed the performance issue in a composition-varying channel by introducing the concept of frequency switching based on the predicted composition of the channel at any given time slot. A more fundamental way to address this problem would be to develop new propagation models that would consider the composition as a stochastic variable in the model. How to accommodate this in a way that does not increase the complexity of the model, yet yields accurate performance for realistic composition-varying channels, such as the ones discussed in this paper, is a challenging problem worth investigating as a future work. 


\section{ACKNOWLEDGMENTS}

The authors acknowledge the use of the Leonardi High Performance Computing resource in the Faculty of Engineering, University of New South Wales for this work.

\section{REFERENCES}

[1] I. F. Akyildiz and J. M. Jornet, "Electromagnetic wireless nanosensor networks," Nano Communication Networks, vol. 1, no. 1, pp. 3-19, Mar. 2010.

[2] J. Jornet and I. Akyildiz, "Graphene-based nano-antennas for electromagnetic nanocommunications in the terahertz band," Proceedings of the Fourth European Conference on on Antennas and Propagation (EuCAP), April 2010.

[3] I. T. Javed and I. H. Naqvi, "Frequency band selection and channel modeling for WNSN applications using simplenano," in Proceeding of the 2013 IEEE International Conference on Communications (ICC). Ieee, Jun. 2013, pp. 5732-5736.

[4] I. F. Akyildiz, F. Brunetti, and C. Blázquez, "Nanonetworks: A new communication paradigm," Computer Networks, vol. 52, no. 12, pp. 2260-2279, Aug. 2008.

[5] J. Villatoro and D. Monzón-Hernández, "Fast detection of hydrogen with nano fiber tapers coated with ultra thin palladium layers." Optics express, vol. 13, no. 13, pp. 5087-92, Jun. 2005.

[6] C. R. Yonzon, D. a. Stuart, X. Zhang, and A. D. McFarland, "Towards advanced chemical and biological nanosensors-An overview." Talanta, vol. 67, no. 3, pp. 438-48, Sep. 2005.

[7] C. Falconi, a. Damico, and Z. Wang, "Wireless Joule nanoheaters," Sensors and Actuators, vol. 127, no. 1, pp. 54-62, Oct. 2007.

[8] E. Zarepour, A. A. Adesina, M. Hassan, and C. T. Chou, "Nano Sensor Networks for Tailored Operation of Highly Efficient Gas-ToLiquid Fuels Catalysts," in the proceedings of Australasian Chemical Engineering Conference, Chemeca, Brisbane, Australia, Oct . 2013.

[9] E. Zarepour, M. Hassan, C. T. Chou, and A. A. Adesina, "Nano-scale Sensor Networks for Chemical Catalysis," in the proceedings of the 13th IEEE International Conference on Nanotechnology, Beijing, China, Aug. 2013.

[10] J. Jornet and I. Akyildiz, "Channel modeling and capacity analysis for electromagnetic wireless nanonetworks in the terahertz band," IEEE Transactions on Wireless Communications, vol. 10, no. 10, pp. 32113221, 2011.

[11] Y. L. Babikov, I. E. Gordon, and S. N. Mikhailenko, “ "HITRAN on the Web ", a new tool for HITRAN spectroscopic data manipulation," in The proceeding of the ASA-HITRAN Conference, Reims, FRANCE, 2012. [Online]. Available: http://hitran.iao.ru/

[12] A. Adesina, "Hydrocarbon synthesis via Fischer-Tropsch reaction: travails and triumphs," Applied Catalysis A: General, vol. 138, no. 2, pp. 345-367, May 1996.

[13] D. Gillespie, "Exact stochastic simulation of coupled chemical reactions," The journal of physical chemistry, vol. 81, no. 25, pp. 2340-2361, 1977.

[14] M.-J. Cros, "Markov Decision Processes (MDP) Toolbox for MATLAB," 2012. [Online]. Available: http://www.mathworks.com.au/matlabcentral/fileexchange/25786markov-decision-processes-mdp-toolbox

[15] J. Jornet and I. F. Akyildiz, "Information capacity of pulse-based Wireless Nanosensor Networks," in Proceeding of the 2011 8th Annual IEEE Communications Society Conference on Sensor, Mesh and Ad Hoc Communications and Networks, Salt Lake, UT, Jun. 2011, pp. 80-88.

[16] J. Zander, "Adaptive frequency hopping in HF communications," in Proceedings of the Military Communications Conference, 1993. MILCOM '93., Boston, USA, 1993, pp. 600-604.

[17] T. Matsumura and K. Mizutani, "Frequency hopping ultra wideband inter-vehicle radar system using chirp waveforms," in Proceeding of the 2004 Ultra Wideband Systems conference, 2004, pp. 386-390.

[18] C.-y. Chen, S. Member, and P. P. Vaidyanathan, "MIMO Radar Ambiguity Properties and Optimization Using Frequency-Hopping Waveforms," IEEE Transactions on signal processing, vol. 56, no. 12, pp. 5926-5936, 2008.

[19] W. C. Chew and J. H. Lin, "A Frequency-Hopping Approach for Microwave Imaging of Large Inhomogeineous Bodies," IEEE Microwave and guided wave letters, vol. 5, no. 12, pp. 439-441, 1995.
[20] M. K. Simon, J. K. Omura, R. A. Scholtz, and B. K. Levitt, Spread Spectrum Communications Handbook, 2nd ed. USA: McGraw-Hill, Inc, 1994.

[21] O. Bamahdi and S. Zummo, "An Adaptive Frequency Hopping TechniqueWith Application to Bluetooth-WLAN Coexistence," in Proceedings of the International Conference on Networking, International Conference on Systems and International Conference on Mobile Communications and Learning Technologies (ICNICONSMCL06), 2006, pp. 1-5.

[22] C. Ding, Y. Yang, and X. Tang, "Optimal Sets of Frequency Hopping Sequences From Linear Cyclic Codes," IEEE Transactions on Information Theory, vol. 56, no. 7, pp. 3605-3612, Jul. 2010.

[23] J.-H. Chung, Y. K. Han, and K. Yang, "New Classes of Optimal Frequency-Hopping Sequences by Interleaving Techniques," IEEE Transactions on Information Theory, vol. 55, no. 12, pp. 5783-5791, Dec. 2009.

[24] D. Gillespie, "A rigorous derivation of the chemical master equation," Physica A: Statistical Mechanics and its Applications, vol. 188, pp. 404 $425,1992$.

\section{APPENDIX}

Modelling a simple chemical process by Markov chain

The paper [24] proves that the dynamics of a set of chemical reactions can be modelled by a continuous-time Markov chain (CTMC). We use a simple example to illustrate the idea. In particular, we want to show how the states and transition probabilities are defined. We

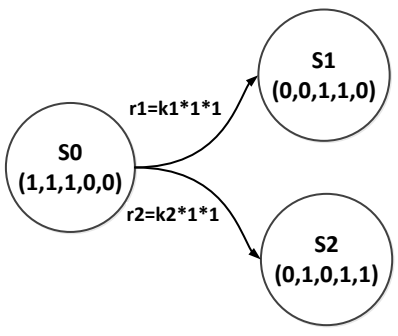

Fig. 9. CTMC of a simple set of chemical reactions.

consider a chemical reactor with 3 input chemical species $A, B$ and $C$. Two possible chemical reactions can take place in this reactor. In the first reaction, a molecule of $A$ reacts with a molecule of $B$ to form a molecule of $D$; this is commonly expressed using the chemical formula of $\mathrm{A}+\mathrm{B} \longrightarrow \mathrm{D}$. The second reaction is $\mathrm{A}+\mathrm{C} \longrightarrow \mathrm{E}$. We use $n_{X}$ to denote the number of molecules of chemical species $X$. The state of the chemical reactor is the 5-tuple $\left(n_{A}, n_{B}, n_{C}, n_{D}, n_{E}\right)$. That is, the state consists of the number of each type of molecules in the chemical reactor. For simplicity, we assume the initial state of the reactor is $S_{0}=(1,1,1,0,0)$ which means there is a molecule of $A$, $B$ and $C$ and no molecules of $D$ and $E$. If the reaction $\mathrm{A}+\mathrm{B} \longrightarrow \mathrm{D}$ occurs, then the state will transit from $S_{0}$ to $S_{1}=(0,0,1,1,0)$ with one molecule of $C$ and $D$. Similarly, if $\mathrm{A}+\mathrm{C} \longrightarrow \mathrm{E}$ occurs, then the state will become $S_{2}=(0,1,0,0,1)$. Given the initial state $S_{0}$, it can be seen that $S_{0}, S_{1}$ and $S_{2}$ are all the allowable states. These are the states of the CTMC, see figure 9.

The transition rate of the CTMC is governed by the rate of chemical reactions. For reaction $\mathrm{A}+\mathrm{B} \longrightarrow \mathrm{D}$, the reaction rate is $r_{1}=k_{1} n_{A} n_{B}$ where $k_{1}$ is the reaction constant of this reaction and a larger $k_{1}$ means a higher likelihood for the reaction to occur (= high state transition rate). Note also that the reaction rate depends on the state. The transition rate from $S_{0}$ to $S_{1}$ is $r_{1}$. Similarly, the transition rate from $S_{0}$ to $S_{2}$ is $r_{2}=k_{2} n_{A} n_{C}$. The CTMC is now completely defined and is illustrated in figure 9 . 